\section{Personalia}

Verleihung der „,Viktor-RuppertMedaille" des Ärzteverbandes Deutscher Allergologen an Herrn Professor Dr. med. Erwin Schöpf

Der Ärzteverband Deutscher Allergologen (ÄDA) hat im Rahmen seines Baden-Württembergischen Fortbildungskongresses in VillingenSchwenningen am 27. Januar 2001 die "Viktor-Ruppert-Medaille" an Herrn Prof. Dr. Erwin Schöpf verliehen. Diese höchste Auszeichnung des Verbandes wurde 1998 vom ÄDA geschaffen, um Persönlichkeiten, die sich in besonderer Weise um die angewandte Allergologie und um den ÄDA verdient gemacht haben, öffentlich zu ehren. Herr Professor Schöpf gehörte seit der Gründung im Jahre 1969 dem Vorstand des ÄDA an und beendete nach 31 Jahren seine Tätigkeit auf eigenen Wunsch im September des vergangenen Jahres. Anlässlich seines Ausscheidens wurde er vom Vorstand zum Ehrenmitglied des Verbandes ernannt. Als Prorektor der AlbertLudwigs-Universität in Freiburg, als Dekan der Medizinischen Fakultät, als Präsident der Deutschen Gesellschaft für Dermatologie, als langjähriges Vorstandsmitglied des ÄDA und in anderen bedeutsamen akademischen und ärztlichen Funktionen hat er in hervorragender Weise sowohl in der wissenschaftlichen Lehre und Forschung wie auch in der allergologischen Weiterbildung zur Qualität und zum Stellenwert dieses Querschnittsfaches beigetragen. In ihm fand die deutsche Allergologie einen Mentor sowohl im Hinblick auf die Interessenvertretung gegenüber den Ministerien und Universitätsgremien wie auch gegenüber den ärztlichen Institutionen. Zu seinen Verdiensten zählt außerdem die Initiierung von nationalen und internationalen Kongressen wie auch von zahlreichen Fortbildungsveranstaltungen zur Qualitätsverbesserung und zur Verbesserung der Patientenversorgung. Die allergologisch tätigen Ärzte in Deutschland sind inm daher zu besonderem Dank verpflichtet.

\section{Dr.J.Wenning}

die Behandlung von Patienten qualitativ verbessert hat und die staatlichen Forschungsanstrengungen intensiviert werden.

Der ALK-SCHERAX Arzneimittel $\mathrm{GmbH}$ sei an dieser Stelle nochmals für die großzügige Unterstützung der Öffentlichkeitsarbeit der Allergologenverbände gedankt.
Dr. rer. nat. Petra von der Lage

Pressekontakt ÄDA/DGAI

MasterMedia $\mathrm{GmbH}$

Bodelschwinghstr. 17

22337 Hamburg

\section{Dr. med. Josef Wenning}

Ärzteverband Deutscher Allergologen Paradiesgasse 2

78050 VS-Villingen

\title{
Fahrtauglichkeit unter Antihistaminika
}

In der ärztlichen Praxis wird immer wieder die Frage auftauchen, ob ein Patient unter seinen ganz individuellen Bedingungen aus Konstitution, Krankheit und Therapie, z.B. mit Antihistaminika, noch fahrtüchtig ist. Wer nicht fahrtüchtig ist, macht sich aber strafbar und verliert u. U. den Versicherungsschutz.

eder Fahrer ist gesetzlich verpflichtet,

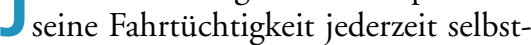
kritisch zu überprüfen. Dies gilt besonders bei Krankheit, Medikamenteneinnahme, Behinderung und höherem Alter. Ob unter der Einnahme eines Antihistaminikums Fahrtauglichkeit besteht, kann jetzt objektiviert werden. Das conTest-Zentrum für Psychometrie (TÜV Kraftfahrt GmbH) in Köln bietet die Untersuchung und Beurteilung der Fahrtauglichkeit auf freiwilliger und vertraulicher Basis an. Die Untersuchung gibt Auskunft, ob das Leistungsvermögen ausreicht, sie dokumentiert die gesetzlich geforderte Sorgfalt des Ratsuchenden und kann auch bedarfsweise bei den Straßenverkehrsbehörden vorgelegt werden.

Auch der Arzt kann im Schadensfall haftbar gemacht werden. Er ist aus verschiedenen Rechtsgründen verpflichtet im Rahmen seiner Behandlung, den Patienten zur Beeinträchtigung der Fahrtauglichkeit konkret aufzuklären und Verhaltensregeln zu geben.

Die Untersuchung dauert etwa drei Stunden. Sie besteht aus sechs Leistungstests (laufen unter dem Oberbegriff „Reaktionstest"): Reaktionsvermögen, Konzentration, Aufmerksamkeit, Motorik, Wahrnehmung, Vigilanz) und verschiedene Sehtests (u.a. Perimetrie, Sehen bei Dunkelheit und Blendung). Nur der

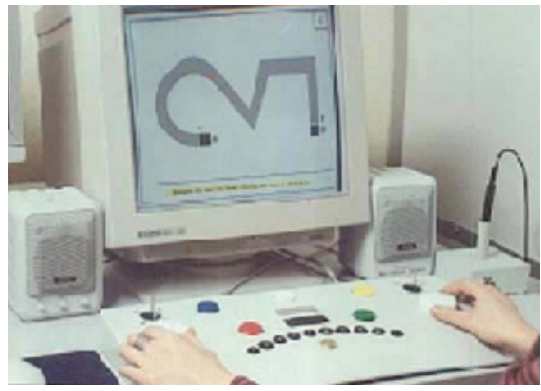

Beim TÜV erfährt der Allergiker, ob er unter Antihistaminika Auto fahren kann.

Patient erhält das schriftliche Gutachten, über dessen weitere Verwendung er allein entscheidet. Die Kosten belaufen sich auf ca. 260 DM (inkl. MWSt.).

Weitere Information und Anmeldung conTest - Zentrum für Psychometrie, Siegburger Str. 231, D-50679 Köln-Deutz, Tel.: 0221 / 650 35-2 22, Fax: 0221 / 650 35-2 50, Internet: www.tuev-contest.de

Impressum „Der ÄDA informiert"

Verantwortlich für den Inhalt: Prof. Dr. Thomas Fuchs

Redaktion: Dr. Corinna K. Leitnaker Urban \& Vogel Medien und Medizin Verlagsgesellschaft mbH \& Co. KG, Neumarkter Straße 43 81637 München 